\title{
An Integrated Approach for Cardiorenal Management
}

Cardiorenal syndrome (CRS) is an intriguing and old pathology that illustrates the paradigm of kidney-heart cross-talk dysfunction. Cardiac and renal dysfunctions may either occur simultaneously because they share causes and pathogenic mechanisms, or sequentially (cardiorenal or renocardiac) because the organ that is primarily affected induces secondary dysfunction and potential tissue damage of the second one. Treatment of CRS has been recently revisited by a number of experts and has benefited from new therapeutic approaches.

In this Blood Purification supplement, we provide an updated pathophysiology of CRS as well as an integrated approach for managing this disorder. In five concise chapters, the 'state of the art' of cardiorenal management practices in acutely decompensated heart patients is presented:

- Peter McCullough and colleagues revisit the pathophysiology of CRS and provide an executive summary of the 11th Consensus Conference of the Acute Dialysis Quality Initiative (ADQI) group.

- Lui Forni and Lakhmir Chawla provide a comprehensive review on the meaning and clinical utility of biomarkers used in CRS.
- Francois Roubille and colleagues deliver a concise systematic review of pharmacologic therapies for chronic and acute decompensated heart failure with specific insights on CRS.

- Huijuan Mao and colleagues reassess factors implicated in cardiac surgery-associated acute kidney injury and therapeutic strategies to prevent this form of CRS.

- Bernard Canaud and colleagues revisit rationale and evidence-based medicine for treating congestive heart failure with ultrafiltration based on the findings of the latest studies.

Although clinical guidelines for managing chronic heart failure are available, best clinical practices concerning the treatment of patients with CRS are still lacking. This practical gap represents an opportunity to offer clinicians a comprehensive yet concise summary of patients affected by CRS. We thank all of the contributors for their excellent contributions. We hope this supplement contributes towards improving the daily care and reducing the burden of CRS.

Bernard Canaud, Bad Homburg/Montpellier Ciro Tetta, Bad Homburg

Peter A. McCullough, Dallas, Tex./Plano, Tex. Lakhmir S. Chawla, Washington, D.C. 